\title{
LES of Reacting Mixing Layers: Species Concentration Boundedness and Inflow Conditions
}

\author{
Anand Kartha*, Pramod K. Subbareddy ${ }^{\dagger}$, Graham V. Candler $^{\ddagger}$, \\ Aerospace Engineering and Mechanics, University of Minnesota, Minneapolis MN 55455, USA \\ Paul E. Dimotakis ${ }^{\S}$ \\ California Institute of Technology, Pasadena, CA 91125, USA
}

\begin{abstract}
The present work carries out large-eddy simulations of the low-speed, high-Reynolds number, chemically-reacting mixing layer experiments by Slessor et al. ${ }^{1}$ In particular, we study the low-heat release case with prescribed turbulent inflow conditions. The objective of the present work is to gain insight into the physics of the reacting shear layer and to address some associated computational challenges. This set of experiments are at subsonic conditions and use hydrogen and fluorine as the fuel and oxidizer, respectively. The hypergolic reaction between $\mathrm{H}_{2}$ and $\mathrm{F}_{2}$, as it was run in the Slessor et al. experiments, is characterized by a large Damköhler number, making the chemistry fast compared to the flow time scales: the product formation and temperature-rise in the flow is mixing-limited. In this work, we attempt to address the issue of overshoots and undershoots of species mass-fractions, often observed in LES of high-Reynolds number flows, by modifying the convective fluxes. We observe that the modified fluxes eliminate the global excursions of species mass-fraction concentration. A three dimensional simulation is performed by imposing synthetic turbulence at the inflow, generated using the digital filter approach of Klein et al., ${ }^{2}$ to mimic the experimental flow conditions. The velocity profiles, growth rate, and product thickness obtained from the simulations show a good match with the experimental data, but the peak value of temperature-rise is slightly over predicted.
\end{abstract}

\section{Introduction}

Mixing of fuel and oxidizer is an important process in scramjet engines. In the combustor section of these engines, high-speed air (oxidizer) is mixed with fuel, leading to chemical reactions and subsequent heat release. To have sustained and efficient combustion, the fuel and the oxidizer should be well-mixed at the molecular level. This necessitates a fundamental understanding of the physics of mixing in turbulent, reacting flows. Since mixing layer geometries are usually simple, they are good candidates for fundamental physics investigations over a wide range of flow conditions. Experimental studies on mixing layers have shown that the inflow boundary states (laminar, transitional or turbulent) of the streams has a significant effect on the molecular-mixing process. ${ }^{1,3-6}$ In this paper, we use LES to study the high-Reynolds number, reacting shear layer at conditions that match the experiments of Slessor et al. ${ }^{1}$

Accurate numerical calculations at these conditions pose several numerical challenges. These nonpremixed flows exhibit over- and under-shoots in the species mass fraction fields in regions of large gradients of species concentrations. This is also seen in conserved passive scalar fields and was investigated in recent studies by Matheou \& Dimotakis ${ }^{7}$ and Subbareddy et al. ${ }^{8}$ Excursions in the species concentration yield aphysical predictions of temperature (and other quantities) in reacting flows. This was observed in simulations of the flow under consideration in a recent paper by Ferrero et al. ${ }^{9}$ Hence, it is of great importance to ensure that numerically computed species concentrations remain bounded.

Reproducing the experimental study of Slessor et al. requires that the boundary conditions match those in the experiments as closely as possible. Mixing layers are sensitive to initial and boundary conditions:

\footnotetext{
*Graduate Research Assistant, Student Member AIAA.

$\dagger$ Research Associate, Member, AIAA.

$\ddagger$ Professor, Fellow, AIAA.

$\S$ Professor, Fellow, AIAA.
} 
these effects persist for a long distance downstream of the splitter plate, influencing the growth of the mixing layers. ${ }^{4,10,11}$ Bell \& Mehta ${ }^{12}$ observed that the presence of streamwise vortices in a laminar-inflow mixing layer increases the entrainment leading to higher growth rates. Some studies ${ }^{12-15}$ have noted that mixing layers that originate from turbulent boundary layers at the inflow grow at a slower rate than those with laminar inflow streams. It has been suggested that the smaller scales associated with turbulent inflow boundary layers inhibit large scale interactions and are responsible for the initial reduction in the growth rate for tripped mixing layers. ${ }^{14}$ Tripping of the incoming boundary layer to a fully turbulent state affects the entrainment rates of the fuel and oxidizer streams into the mixing region. This gives rise to a specific species composition profile and a so-called 'marching' probability distribution function for the scalar fields.

There are a few previous computational studies on the effect of inflow conditions on mixing layers. Direct numerical simulations (DNS) of temporal mixing layers have studied the effect of initializing the flow with low and high gradient laminar boundary layers and with two and three dimensional perturbations. ${ }^{16,17}$ The use of synthetic turbulence to model a turbulent inflow for a spatially evolving mixing layer has also been studied. ${ }^{18,19}$ More recently McMullan \& Garrett ${ }^{20}$ used artificially generated inflow turbulence to investigate the effect of initial conditions for a low-speed mixing layer. However, a numerical study of the effect of inflow turbulence on a reacting, spatially evolving mixing layer has not been done, to the best of our knowledge.

The experimental study this paper aims to reproduce were performed by Slessor et al.: ${ }^{1}$ the aim was to examine the effect of inflow conditions on a reacting mixing layer. In these experiments, hydrogen (fuel) and fluorine (oxidizer) are present in small amounts in the faster moving (top) and slower moving (bottom) streams, respectively. Heat release due to the hypergolic reaction between hydrogen and fluorine at the conditions studied gives an adiabatic flame temperature rise of $171 \mathrm{~K}$. The effect of inflow conditions was analyzed via the temperature rise measured at several probe locations. They observed that changing the inflow conditions by tripping the incoming boundary layers reduces the entrainment and shear layer growth rate far downstream of the splitter plate. Intense stirring and effective molecular mixing was observed in the experiments with the tripped boundary layer. A decrease in the two-dimensional organization of the vortex structures was also seen. However, only small changes were noted in the mean streamwise velocity along the transverse direction.

\section{Numerical method and computational setup}

\section{Governing equations and numerical method}

We solve the LES equations obtained by spatially filtering the equations for conservation of mass (separate equations are solved for each of the species densities), momentum and energy. Using the notation $\widetilde{(\cdot)}$ to denote Favre averaging $(\tilde{f}=\overline{\rho f} / \bar{\rho})$, the filtered equations are

$$
\begin{gathered}
\frac{\partial \bar{\rho}_{s}}{\partial t}+\frac{\partial \bar{\rho}_{s} \tilde{u}_{j}}{\partial x_{j}}=-\frac{\partial \bar{\rho}_{s} \tilde{v}_{s, j}}{\partial x_{j}}-\frac{\partial \mathcal{V}_{s, j}}{\partial x_{j}}+\bar{\omega}_{s} \\
\frac{\partial \bar{\rho} \tilde{u}_{i}}{\partial t}+\frac{\partial \bar{\rho} \tilde{u}_{i} \tilde{u}_{j}}{\partial x_{j}}=-\frac{\partial \bar{p}}{\partial x_{i}}+\frac{\partial \bar{\sigma}_{i j}}{\partial x_{j}}-\frac{\partial \tau_{i j}}{\partial x_{j}} \\
\frac{\partial \bar{E}}{\partial t}+\frac{\partial}{\partial x_{j}}\left[(\bar{E}+\bar{p}) \tilde{u}_{j}+\bar{q}_{j}-\bar{\sigma}_{i j} \tilde{u}_{i}+\sum_{s} \bar{\rho}_{s} \tilde{h}_{s} \tilde{v}_{s, j}\right]=-\frac{\partial}{\partial x_{j}}\left(q_{t, j}+\mathcal{J}_{j}-\mathcal{D}_{j}+\sum_{s} h_{s}^{o} \mathcal{V}_{s, j}\right) .
\end{gathered}
$$

In the above equations, the species densities are $\rho_{s} \equiv \rho c_{s}$, where $c_{s}$ is the mass fraction of species $s$. The filtered pressure, total energy, viscous shear stress, and heat flux vector are

$\bar{p}=\sum_{s} \bar{\rho}_{s} \frac{R}{M_{s}} \tilde{T}, \quad \bar{E}=\sum_{s} \bar{\rho}_{s} c_{v, s} \tilde{T}+\frac{1}{2} \bar{\rho} \tilde{u}_{k} \tilde{u}_{k}+\sum_{s} \tilde{\rho}_{s} h_{s}^{o}+\bar{\rho} k, \quad \bar{\sigma}_{i j}=2 \mu\left(\tilde{S}_{i j}-\frac{1}{3} \tilde{S}_{k k} \delta_{i j}\right), \quad \bar{q}_{j}=-\kappa \frac{\partial \tilde{T}}{\partial x_{j}}$,

where $c_{v, s}$ and $c_{p, s}$ are the species specific heats at constant volume and pressure, respectively. $\tilde{S}_{i j}$ is the symmetric part of the velocity gradient tensor, $\mu$ and $\kappa$ are the dynamic viscosity and thermal conductivity coefficients, $R$ is the universal gas constant and $M_{s}$ is the molecular weight of species $s$. The species mass diffusion flux is assumed to follow Fick's law,

$$
\bar{\rho}_{s} \tilde{v}_{s, j}=-\bar{\rho} D \frac{\partial \widetilde{c_{s}}}{\partial x_{j}}
$$


where, in this paper, a single binary diffusion coefficient, $D$, derived assuming a constant Lewis number $(\mathrm{Le}=1.4)$, is used in the simulations. The source term due to chemical reactions is $\bar{\omega}_{s}$ and the heat of formation of each species is $h_{s}^{\circ}$. The species enthalpy is defined as $\tilde{h}_{s}=c_{p, s} \tilde{T}+h_{s}^{\circ}$.

The sub-grid scale (SGS) terms that need to be modeled are the SGS stress tensor, $\tau_{i j}$, the turbulent mass flux, $\mathcal{V}_{s, j}$, the turbulent heat flux, $q_{t, j}$, the turbulent diffusion $\mathcal{J}_{j}$, and the SGS viscous diffusion $D_{j}$. In this work, $\mathcal{J}_{j}$ and $\mathcal{D}_{j}$ are ignored. $\mathcal{V}_{s, j}, \tau_{i j}$ and $q_{t, j}$ are modeled using an eddy-viscosity assumption

$$
\mathcal{V}_{s, j}=-\frac{\mu_{t}}{\mathrm{Sc}_{\mathrm{t}}} \frac{\partial \tilde{c}_{s}}{\partial x_{j}}, \quad \tau_{i j}=-2 \mu_{t}\left(\tilde{S}_{i j}-\frac{1}{3} \tilde{S}_{k k} \delta_{i j}\right), \quad q_{t, j}=-\frac{\mu_{t}}{\operatorname{Pr}_{\mathrm{t}}} \frac{\partial \tilde{T}}{\partial x_{j}},
$$

in which $\tilde{S}_{i j}$ is the resolved rate of strain tensor and the turbulent Schmidt $\left(\mathrm{Sc}_{\mathrm{t}}\right)$ and Prandtl $\left(\mathrm{Pr}_{\mathrm{t}}\right)$ numbers are constants of the model. The values of $\mathrm{Sc}_{\mathrm{t}}$ and $\mathrm{Pr}_{\mathrm{t}}$ used in this work are 0.33 and 0.9 respectively. In this work, the turbulent kinetic energy, $\bar{\rho} k$, is ignored.

The source term $\bar{\omega}_{s}=\bar{\omega}_{s}\left(c_{s}, T\right)$ is a nonlinear function of the species concentrations and the temperature. In the present LES, this term is simply computed as

$$
\overline{\omega_{s}\left(c_{s}, T\right)} \approx \omega_{s}\left(\tilde{c}_{s}, \tilde{T}\right)
$$

which means that the effects of SGS turbulent fluctuations on the chemical source terms are neglected.

The simulations are performed using US3D, a finite-volume, unstructured-grid fluid dynamics code that solves the compressible Navier-Stokes equations. ${ }^{21}$ The inviscid fluxes are evaluated using a blend of the central, non-dissipative, kinetic-energy consistent fluxes of Subbareddy and Candler, ${ }^{22}$ extended to higher order on smoothly varying hexahedral meshes, ${ }^{23}$ and the dissipative portion of an upwind-biased TVD scheme based on flux vector splitting,

$$
F_{\text {inviscid }}=F_{\text {central }}+\alpha F_{\text {diss }}
$$

where $\alpha \in[0,1]$ is a shock sensor due to $\operatorname{Ducros}^{24}$ et al. In the present work, 4th-order inviscid fluxes are used. Viscous fluxes are evaluated using a second-order central scheme. The one-equation Spalart-Allmaras (SA) model is used for turbulence closure. Although SA was originally developed as a RANS model, in this work we use it in its DES form with minor modifications: the wall functions are disabled by setting $f_{v 1}=1$, $f_{v 2}=0$ and $f_{w}$ is set to its asymptotic value of $f_{w}^{*}=0.424$. Both the high-order convective fluxes and the viscous fluxes require the gradients of fluid variables: these are computed using a weighted least-squares method. ${ }^{25}$ The third order, 'strong-stability-preserving' explicit Runge-Kutta scheme (Shu and Osher ${ }^{26}$ ) is used to advance the simulation in time.

\section{Scalar boundedness}

Simulations involving transport of multiple species (or scalar fields, in general) are often subject to spurious overshoots in the species mass fractions leading to issues with conservation of mass: this is usually the case if species concentrations are not properly upwinded in the regions where sharp gradients in species concentration are present. Similar overshoots have been observed in computations of passive scalar fields and the issue has been recently addressed in work by Zhang and Shu. ${ }^{27}$ We use ideas from their paper and limit the reconstructed values of species concentrations. Henceforth, in this paper, the flux scheme with limited species concentrations will be referred to as 'modified fluxes' and the scheme without it will be referred as 'standard fluxes'.

In this work, we follow the procedure from the paper by Subbareddy et al. ${ }^{8}$ to ensure boundedness of species concentrations. For passive scalars, natural bounds (usually between 0 and 1) exist and place clear limits on the allowable concentrations. However, determining similar limits for species concentrations in a reacting flow simulation is non-trivial. In a reacting flow, in addition to changes brought about by mixing, species are formed and consumed; this dynamically changes the local species mass fractions and the global maximum and minimum values for these quantities. To ensure passive scalar boundedness, each of these scalars present in the flow can be treated independently to ensure a meaningful solution. However, for active species, in addition to bounding each species' concentration, there are other constraints that must be satisfied (for e.g., they should sum up to unity). This coupling in the system compounds the difficulty of the problem. In addition, one must ensure that the convective fluxes for the momentum and the energy are consistent with that of the mass flux. 


\section{Reaction mechanism}

The chemical reaction we are concerned with here is between molecular hydrogen and fluorine and can be formally written as

$$
\mathrm{H}_{2}+\mathrm{F}_{2} \rightleftharpoons 2 \mathrm{HF}, \quad \Delta \mathrm{Q}=-544 \mathrm{~kJ} / \text { mole. }
$$

However a more realistic reaction mechanism is composed of the hydrogen-fluorine chain reaction initiated by nitric oxide:

$$
\begin{aligned}
\mathrm{NO}+\mathrm{F}_{2} \stackrel{k_{1}}{\rightleftharpoons} \mathrm{NOF}+\mathrm{F}, & \Delta Q=-76.6 \mathrm{~kJ} / \text { mole }, & k_{1}=4.2 \times 10^{8} \exp \left(\frac{-1150}{T}\right), \\
\mathrm{H}_{2}+\mathrm{F} \stackrel{k_{2}}{\rightleftharpoons} \mathrm{HF}+\mathrm{H}, & \Delta Q=-133.9 \mathrm{~kJ} / \text { mole }, & k_{2}=2.6 \times 10^{9} T^{0.5} \exp \left(\frac{-307}{T}\right), \\
\mathrm{F}_{2}+\mathrm{H} \stackrel{k_{3}}{\rightleftharpoons} \mathrm{HF}+\mathrm{F}, & \Delta Q=-411.3 \mathrm{~kJ} / \text { mole }, & k_{3}=3.0 \times 10^{6} T^{1.5} \exp \left(\frac{-845.5}{T}\right) .
\end{aligned}
$$

Although additional reactions for the formation of NOF and dissociation of $\mathrm{F}_{2}$ could be considered, they are ignored due to the much larger computational cost involved: in previous work by Ferrero et al. ${ }^{9}$ a validation of the chemistry model was presented and it was shown that using the reaction set (6-8) is adequate.

The chemical time, $\tau_{\text {chem }}$ that it takes for the overall reaction set (6-8) to complete is used to define the Damköhler number,

$$
\mathrm{Da}=\frac{\tau_{\text {mix }}}{\tau_{\text {chem }}},
$$

where $\tau_{\text {mix }}$ is the fluid-mechanical mixing time scale. Flows with Da $\gg 1$ are characterized by kineticallyfast chemical reactions and the amount of product formed is limited by the amount of mixing achieved. In this regime, product formation is directly related to the amount of heat released to the system and this property has been exploited in several experimental investigations. From a computational standpoint, however, kinetically-fast reactions pose a challenge if the source term is treated in an explicit manner, as is done in this work. The kinetically-fast reactions impose a strict requirement on the timestep for stable numerical simulation, leading to a physical time step of approximately $1.5 \times 10^{-7} \mathrm{sec}$ for the present calculation.

\section{Computational Setup}

Figure 1 shows a sketch of the experimental facility used by Slessor et al. The portion of the domain used in the computation is highlighted by a rectangle (in red). The heights of the test section above and below the splitter plate are $h_{1}=5 \mathrm{~cm}$ and $h_{2}=10 \mathrm{~cm}$, respectively. The length of the test section, from the inlet to the location of the probes is $L=41.5 \mathrm{~cm}$. The computational domain dimensions are $[0,0.65] \times[-0.06,0.04] \times[0,0.06]$ meters in the streamwise $(x)$, transverse $(y)$ and spanwise $(z)$ directions. The $y=0$ plane coincides with the top surface of the splitter plate. The grid is stretched in the transverse direction and around ten grid elements are used to capture the boundary layer profiles on either side: this is relatively coarse. The finite thickness of the splitter plate tip is not resolved in these simulations. The grid is progressively stretched beyond the probe location (at $x \approx 0.41 \mathrm{~m}$ ). In the experiments, the angle of the lower wall is adjusted ( $\beta \approx 0.5$ degrees) to accommodate the effect of the shear layer growth and to maintain a zero pressure gradient in the streamwise direction. The computational domain contains 180 grid points in the transverse direction and 90 equally spaced points in the spanwise direction, resulting in a total grid size of $\approx 6$ million.

\section{Initial and Boundary Conditions}

The top stream in the present study enters the test section at a velocity of $U_{1} \simeq 100 \mathrm{~m} / \mathrm{s}$ and the bottom stream at $U_{2} \simeq 40 \mathrm{~m} / \mathrm{s}$. The static pressure and temperature at the inlets of the test section for both the streams are close to atmospheric conditions. In the experiments, the effect of the inflow conditions was analyzed by tripping the incoming boundary layers leading to a turbulent inflow stream at the tip of the splitter plate. 


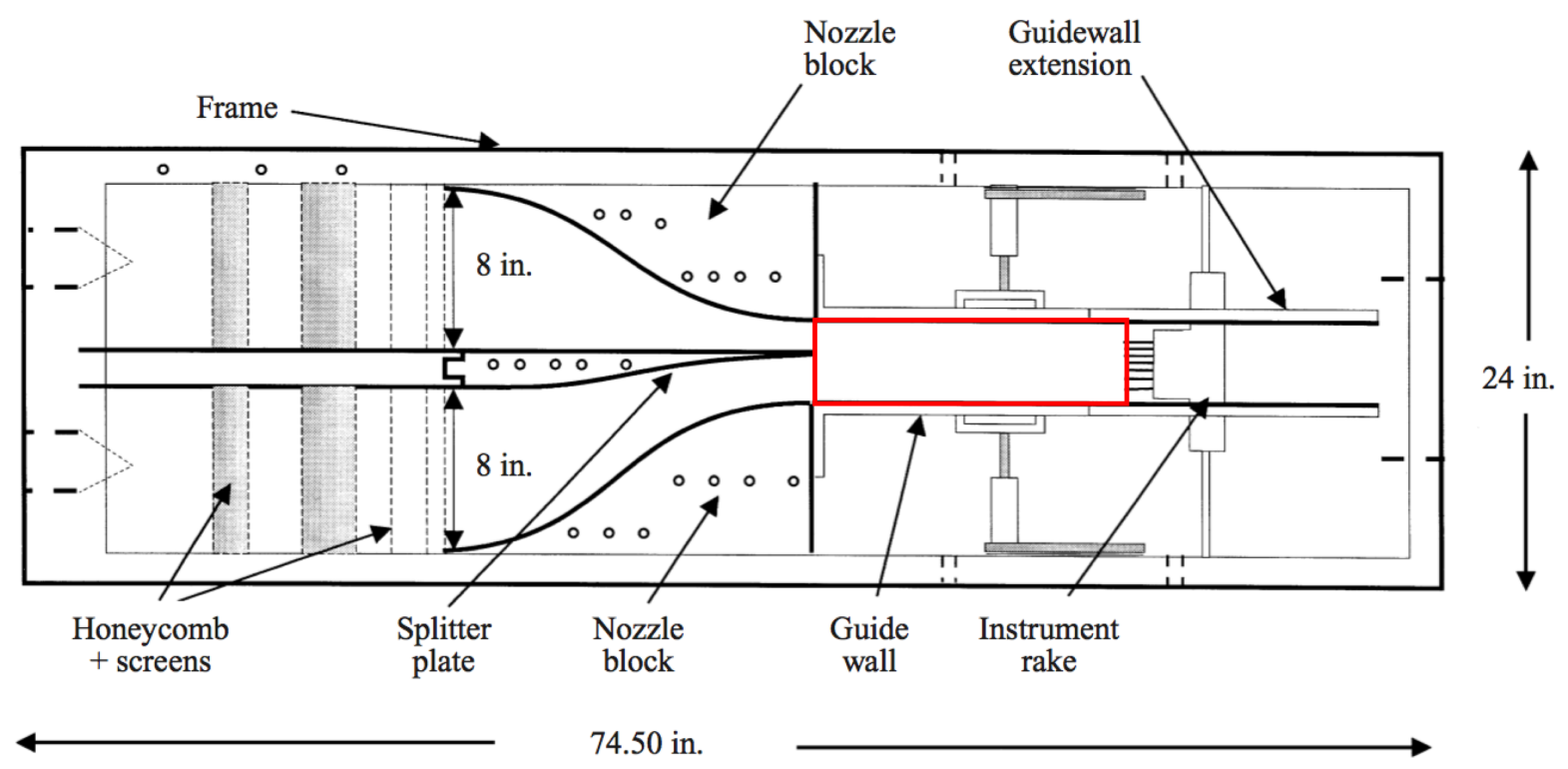

Figure 1. The experimental facility used by Slessor et al. at Caltech. ${ }^{1}$ The red rectangle indicates the test section which is modeled in the simulations.

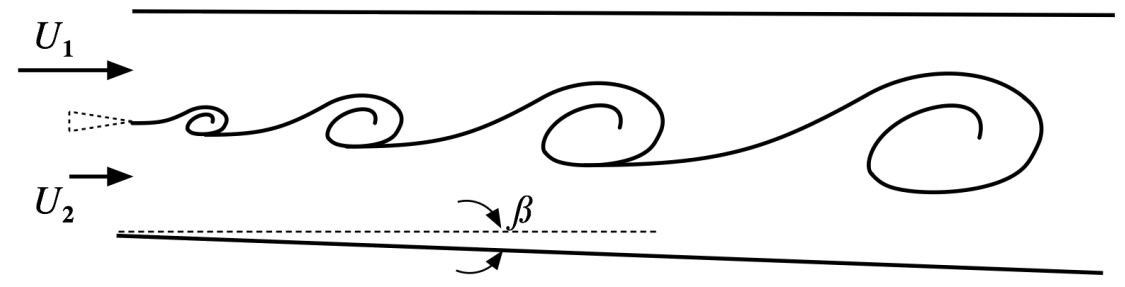

Figure 2. Cartoon of the experimental setup illustrating the inclination on the lower wall. $\beta$ is the angle of the lower wall. 
Table 1. Summary of the flow conditions chosen for the present work. Flow conditions closely match the experiments of Slessor et al. ${ }^{1}$

\begin{tabular}{cllll} 
Run & \multicolumn{2}{c}{ Chemical Composition (mole fractions) } & $\phi$ & $\Delta T_{f}$ \\
\hline \hline & Primary stream & Secondary stream & & \\
\hline Case 1 & $\mathrm{H}_{2}=0.93 \%, \mathrm{NO}=0.14 \%$, & $\mathrm{F}_{2}=8.00 \%, \mathrm{~N}_{2}=76.0 \%$, & 8 & $171 \mathrm{~K}$ \\
(Fluorine rich) & $\mathrm{Ar}=8.00 \%, \mathrm{He}=15.07 \%$, & $\mathrm{He}=16.0 \%$. & & \\
& $\mathrm{N}_{2}=75.86 \%$. & & \\
Case 2 & $\mathrm{Ar}=8.00 \%, \mathrm{He}=16.00 \%$, & $\mathrm{Ar}=8.0 \%, \mathrm{He}=16.00 \%$, & \\
(non-reacting) & $\mathrm{N}_{2}=76.00 \%$. & $\mathrm{N}_{2}=76.00 \%$. & \\
\hline
\end{tabular}

In addition, the effect of heat release and the extent of molecular mixing is analyzed by varying the chemical composition. The compositions for the cases considered here are shown in Table 1.

Since the flow is subsonic, it is subject to spurious numerical reflections at the boundaries unless proper boundary conditions are used. We use characteristic-based boundary conditions (based on Poinsot and Lele ${ }^{28}$ ) at the inflow and outflow boundaries of the domain. Turbulent velocity profiles computed using RANS simulations along with synthetic fluctuations generated using the digital filter technique ${ }^{2,29,30}$ are imposed at the inflow boundaries. This is briefly discussed in the following sub-section. The subsonic outflow boundary conditions are modeled after Poinsot and Lele ${ }^{28}$ and the work of Yoo and Im. ${ }^{31}$ The top and the bottom walls of the test section are treated as slip walls and periodic boundary conditions are enforced in the spanwise direction. In a 'sponge layer' that extends from a location downstream of the experimental probe locations, numerical dissipation is progressively added as a function of streamwise distance to minimize numerical reflections from the outflow boundary.

\section{Generation of inflow turbulence}

We use the digital filter technique (Klein et al., ${ }^{2}$ Xie \& Castro, ${ }^{29}$ Touber \& Sandham ${ }^{30}$ ) to generate the synthetic turbulence field. With this method, inflow perturbations are generated to match prescribed Reynolds stresses. An instantaneous snapshot of the generated inflow turbulence is shown in figures 3 and the time averaged statistics are matched as shown in figures 4 . These fluctuating quantities are added to the mean flow and imposed at the inflow plane of the computational domain. The mean flow profile was taken from a RANS calculation in which a numerical trip is placed at the location where the laminar boundary layer has a momentum thickness of $0.095 \mathrm{~mm}$ to match the flow conditions just before the trip as mentioned in Slessor et al. Dimensions of the numerical trip match the dimension of the trip wire ( $0.8 \mathrm{~mm}$ diameter) used in the experiments.

\section{Species mass fraction behavior}

\section{Temporal mixing layer}

To handle spurious overshoots and undershoots in the species mass fractions, the fluxes were modified, as discussed earlier. To assess the performance of these changes, we use a fairly standard test case: the temporal mixing layer studied by Yee et al. ${ }^{33}$ As a baseline, simulations were first performed with a single species in the flow $\left(\mathrm{N}_{2}\right)$ and with standard (unmodified) fluxes, using conditions that can be found in Yee's paper. The result is shown in figure 5(b). In subsequent simulations, the top stream is initialized with a gas that is composed of $\mathrm{N}_{2}$ and a 'marker' species, $\mathrm{N}_{2}^{\mathrm{A}}$ in equal proportions. The bottom stream is similarly initialized with $\mathrm{N}_{2}$ and a different marker, $\mathrm{N}_{2}^{\mathrm{B}}$. These marker species, $\mathrm{N}_{2}^{\mathrm{A}}$ and $\mathrm{N}_{2}^{\mathrm{B}}$, have the same properties as $\mathrm{N}_{2}$ but are tagged differently to study the effect of sharp gradients in multi-species calculations. The result of the multi-species simulation with modified fluxes, shown in figure 5(c), are very similar to the results from the single species calculation. The results are overlaid in figure 5(d) to directly compare the two solutions. The computation with modified fluxes ensures that the species mass fractions are bounded. Figure 6 shows the maximum values of the marker species $\mathrm{N}_{2}^{\mathrm{A}}$ in the domain as a function of time and clearly displays the 


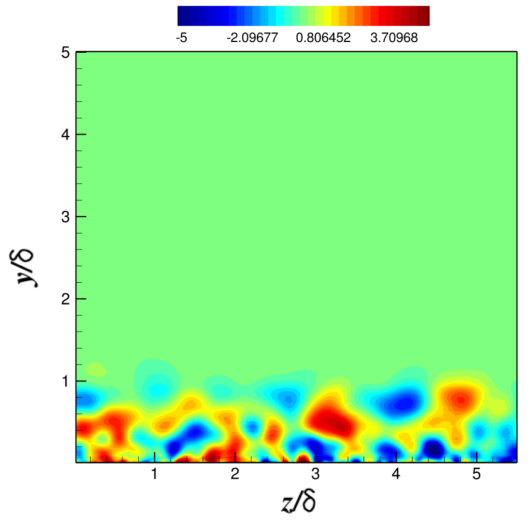

(a)

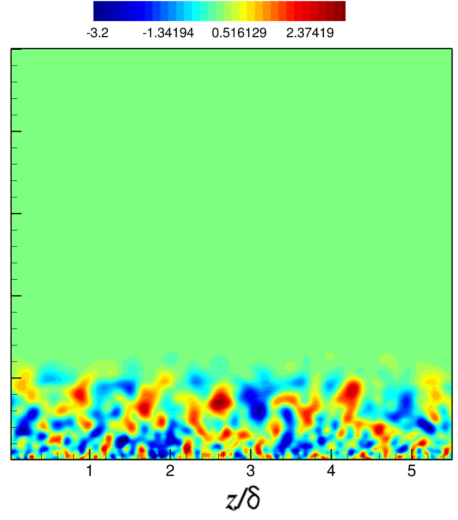

(b)

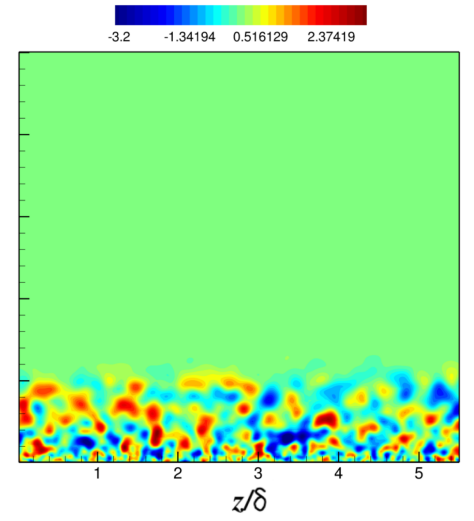

(c)

Figure 3. Contours of instantaneous fluctuating quantities added to the inflow: (a) $u^{\prime} / u_{\tau}$ (b) $v^{\prime} / u_{\tau}(\mathbf{c}) w^{\prime} / u_{\tau}$.

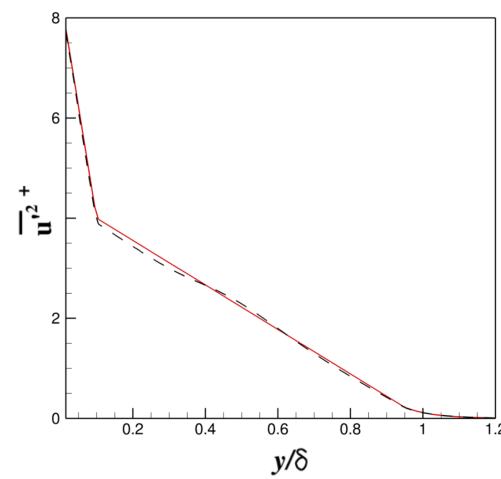

(a)

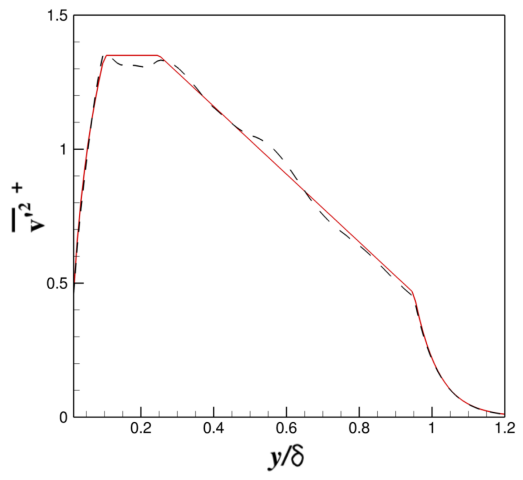

(b)

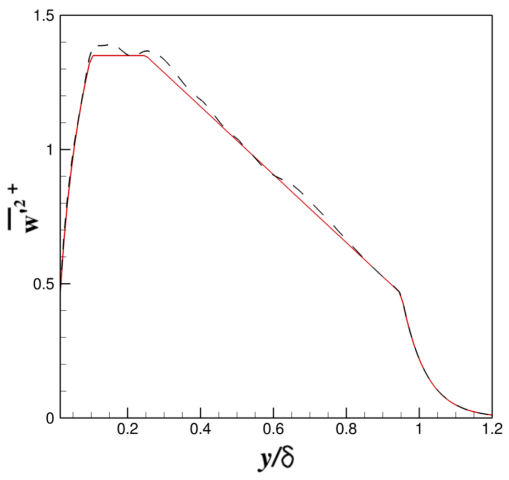

(c)

Figure 4. Comparison of the time-averaged statistics (black-dashed lines), from the generated inflow turbulence with digital filter technique, with (approximate) curve fits to the experimental data (red-solid line) from DeGraaff and Eaton. ${ }^{32}$ 
effectiveness of the new fluxes in bounding the solution. The result of a simulation performed using standard fluxes for the multi-species case is shown in figure 5(e): the disagreement with the canonical result can be clearly seen. We believe that this is due to excursions in species mass fractions $\left(\mathrm{N}_{2}^{\mathrm{A}}\right)$.

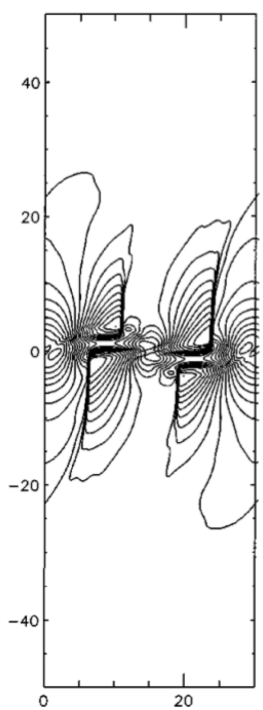

(a)

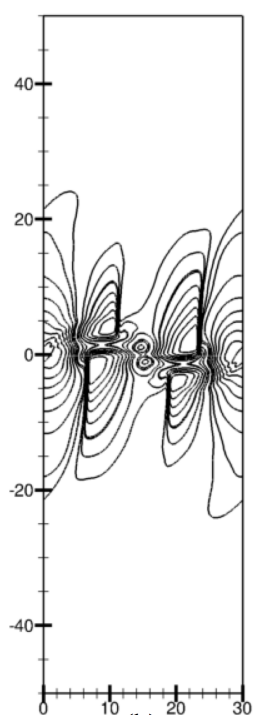

(b)

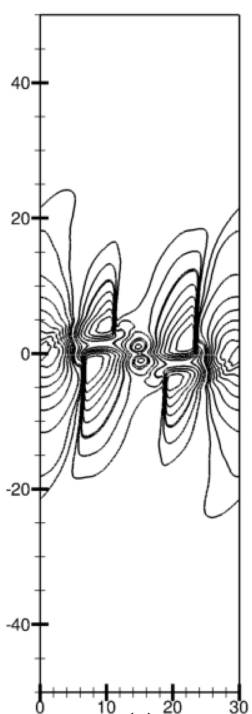

(c)

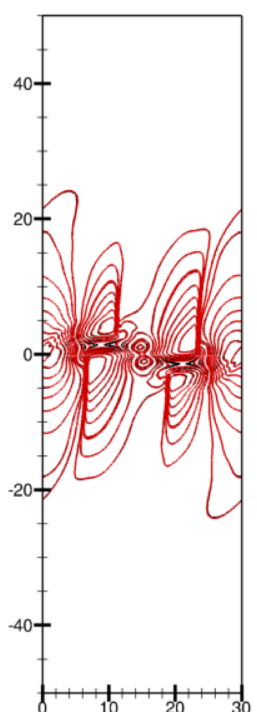

(d)

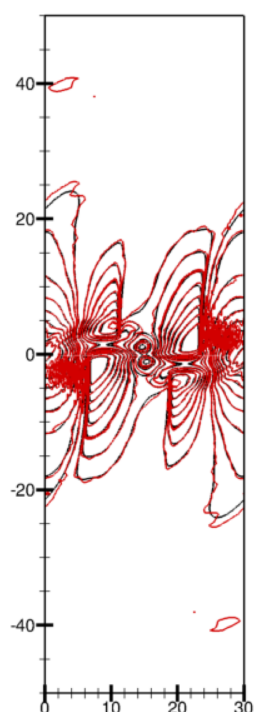

(e)

Figure 5. Comparison of temperature contours obtained from standard and modified fluxes: (a) result from Yee et al. corresponding to a non-dimensional time of $80,(\mathrm{~b})$ contours obtained with standard fluxes for single species, (c) contours obtained with modified fluxes for multiple species, (d) contours from (b) and (c) are overlaid for comparison, (e) contours obtained with standard fluxes for multiple species overlaid with contours obtained in (b).

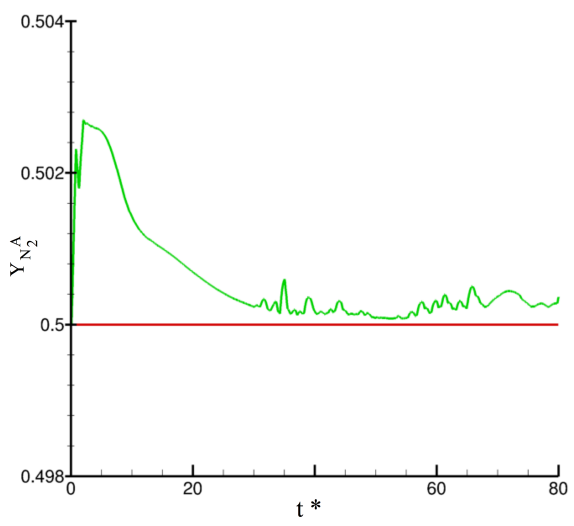

Figure 6. Variation of maximum value of species concentration $\left(\mathrm{N}_{2}^{\mathrm{A}}\right)$ with time. Green line is obtained from simulation with standard fluxes while, the red line is obtained from simulation with modified fluxes.

\section{Two-dimensional spatially developing mixing layer}

In order to further clarify the nature of the scalar excursions and to understand the behavior of the modified fluxes, we next simulated spatially evolving two-dimensional mixing layers at conditions that match the experiments we are studying in this paper. First we simulate a non-reacting case in which argon that is present in both streams is tagged as $\mathrm{Ar}^{\mathrm{A}}$ in the top stream and $\mathrm{as}^{\mathrm{A}} \mathrm{B}^{\mathrm{B}}$ in the bottom stream. Figure 7 (a) clearly highlights regions of scalar overshoots near the edges of the mixing layer. These overshoots 
are absent in figure 7(b) which was obtained with modified fluxes. However, the highly diffused structures in this figure also reveal the excessive levels of dissipation due to the flux modification. To overcome this issue, the numerical dissipation was localized using a switched dissipation function: the results of using the localized switch in addition to the modified fluxes can be seen in figure 7(c). Note the much sharper resolution of the flow features. These fluxes were then used to compute a two-dimensional reacting mixing layer (conditions match case 1 from Table 1). Instantaneous temperature contours from this simulation are shown in figure 8. Note that overshoots in the species mass fractions are absent. However, there are regions in the flow where the temperature goes above the adiabatic flame temperature. As mentioned in Table 1, the adiabatic temperature rise for this case is $171 \mathrm{~K}$ which corresponds to a temperature of $463 \mathrm{~K}$ in the simulations. In figure 8 , white contours mark the regions where the local temperature exceeds the adiabatic flame temperature. While these temperature excursions are significantly lower than that observed by Ferrero et al. ${ }^{9}$ the cause is as yet not fully understood by us and work is underway to clarify the matter. We believe that these temperature hot-spots may be due to small inconsistencies in the computation of the convective fluxes for the energy equation.

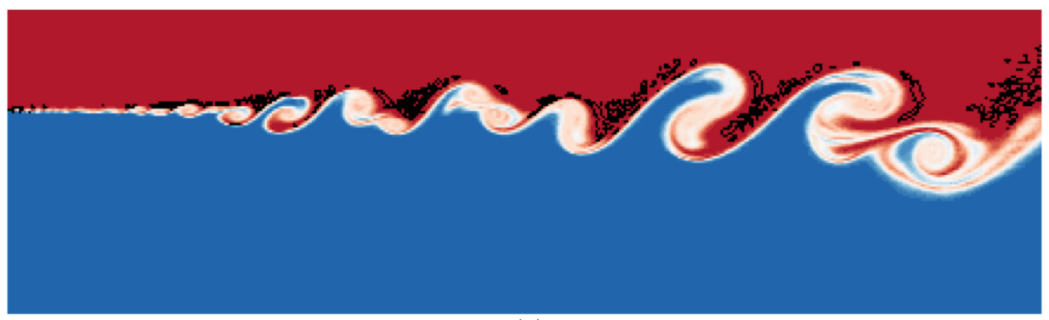

(a)

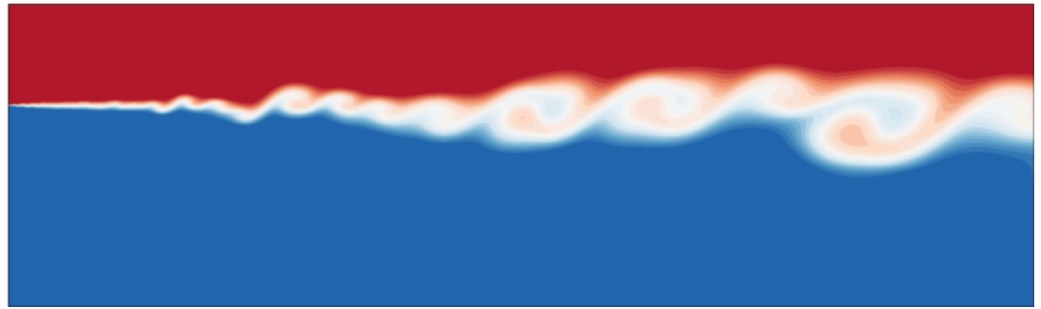

(b)

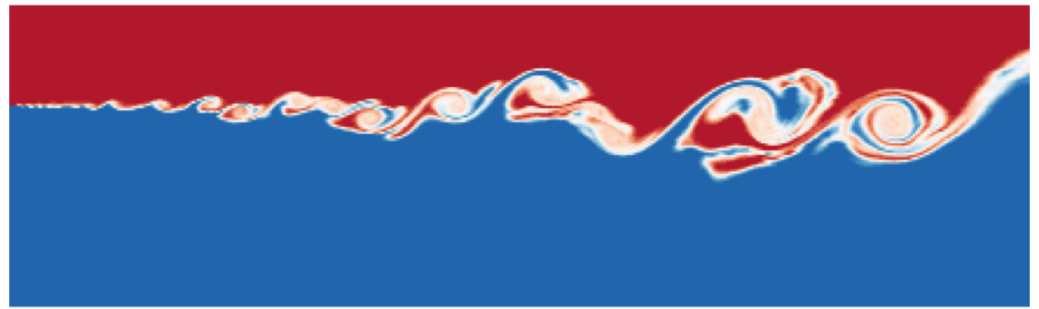

(c)

Figure 7. Comparison of instantaneous contours of $\mathrm{Ar}^{\mathrm{A}}$ concentration (present only in the top stream): (a) using standard fluxes, the black contours show the regions of excursion, (b) using modified fluxes and (c) using modified fluxes with highly local dissipation.

\section{Results}

In this section we discuss results from a three-dimensional simulation at conditions discussed in the previous section and compare with results from the experimental study by Slessor et al. We initially compute a two-dimensional flowfield (this was run for approximately two flow-through times based on distance from the inlet to the measurement-probe locations and convective velocity). The solution from this was used 


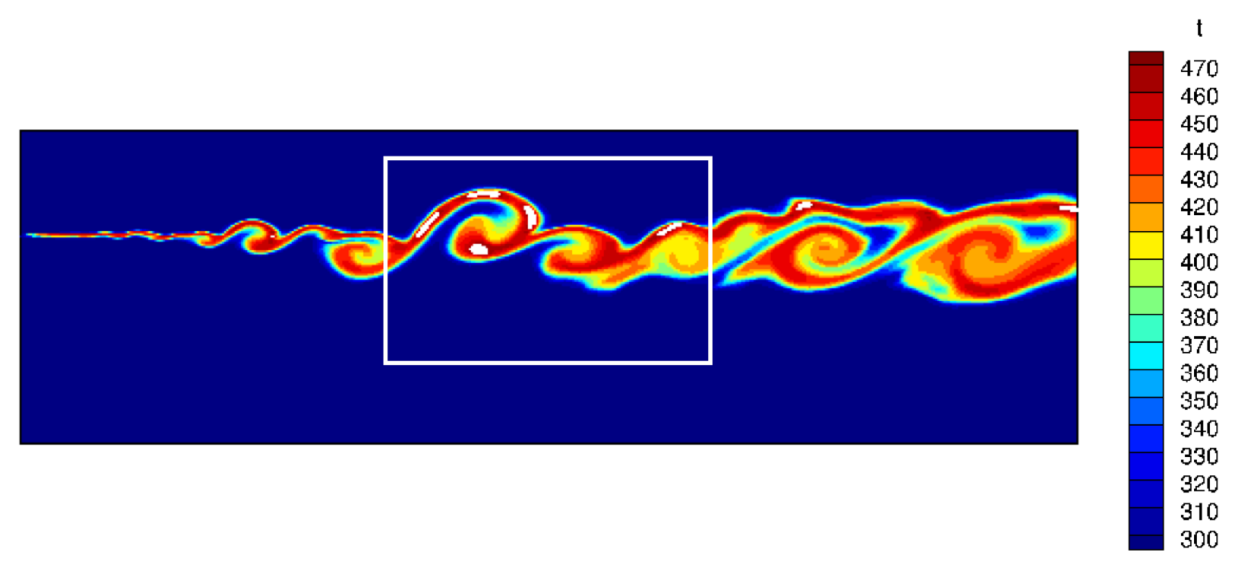

Figure 8. Temperature contours for two-dimensional simulation of Case 1 as mentioned in Table 1. The white contours show the regions where temperature exceed the adiabatic flame temperature.

as an initial condition for the three-dimensional simulation. This considerably lowers the cost associated with allowing the acoustic transients to wash out using the full three-dimension geometry. Then, the three dimensional solutions are performed for at least one flow through time to allow for the flow to switch over from its artificial initial condition. This starting procedure, along with the characteristics-based subsonic boundary conditions ensures that the desired value of pressure is maintained in the computational domain. Synthetic inflow turbulence generated using a digital filter technique is then added to the top stream to mimic the turbulent inflow conditions that was used in the experiments.

\section{Velocity profiles and Reynolds stresses}

Statistics are collected for approximately half a flow through time and averaged in the spanwise direction. The mean profiles are compared with the experimental data collected at a measuring station located at $x=0.415 \mathrm{~m}$ (measured from the inlet of the test section). The mean velocity profile, shown in figure 9 shows excellent agreement with the experimental data. Both the spread and the shape of the velocity profile is well captured. The lower wall angle ensures that the pressure inside the domain is nearly constant: this was critical to get the correct growth rate. Reynolds stress profiles at different locations along the streamwise direction are shown in figure 10. Note that these are normalized using the square of the convective velocity, $U_{c}$, computed using the formula

$$
U_{c}=\frac{a_{2} U_{1}+a_{1} U_{2}}{a_{2}+a_{1}}
$$

where $U_{1}, U_{2}$ are the top and bottom stream velocities and $a_{1}, a_{2}$ are the corresponding speeds of sound. We observe that these plots nearly collapse beyond $x=0.25 \mathrm{~m}$, indicating some level of self similarity beyond this streamwise location.

\section{Growth rate}

The growth of the mixing layer from the simulation is compared with the experimental values in Table 2. The mixing layer thickness $\left(\delta_{T}\right)$ is calculated, as in the experiments, as the distance between the two points where the normalized temperature,

$$
\Theta(y)=\frac{\Delta T}{\Delta T_{a d}},
$$

is at $1 \%$ of the peak value. The growth rate of the mixing layer $\left(\delta_{T} / x\right)$ is evaluated using the above definition and found to be very close to the experimental value. The mixing layer thickness and the chemical product thickness,

$$
\delta_{p}=\int_{-\infty}^{\infty} \Theta(y) d y
$$




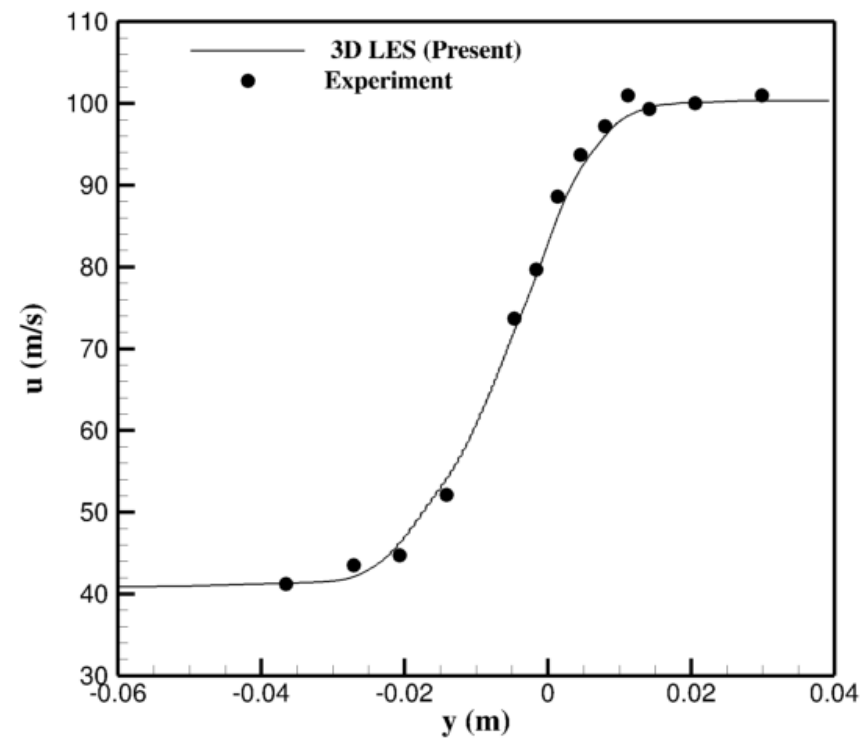

Figure 9. Comparison of velocity profiles from the simulation and experiments, measured at $x=0.415 \mathrm{~m}$.

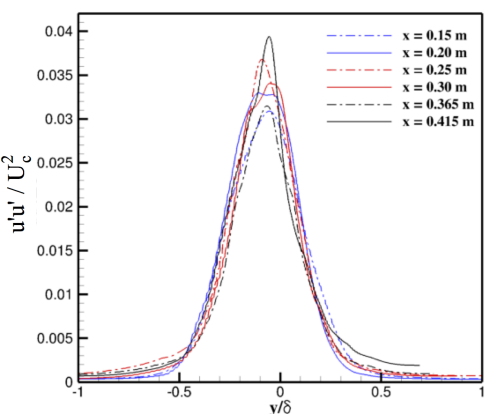

(a)

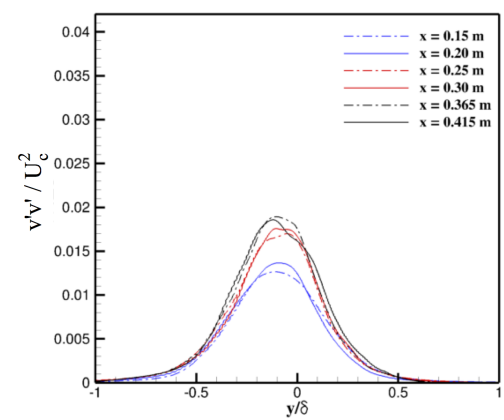

(c)

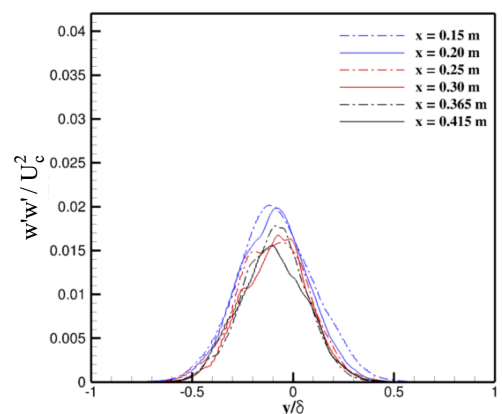

(b)

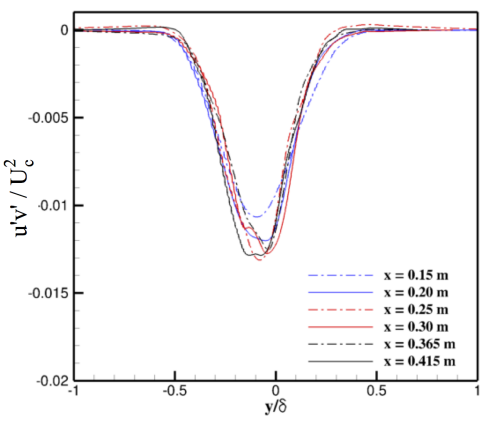

(d)

Figure 10. Normalized Reynolds stress profiles at different streamwise locations. 
are calculated at the measuring station and show reasonable agreement with the experimental value. The non-dimensional product fraction $\left(\delta_{p} / \delta_{T}\right)$, which is a measure of chemical-product formation and is insensitive to the local mixing layer thickness is also predicted reasonably accurately. Instantaneous contours of the density gradient magnitude field (sometimes used as a surrogate for a numerical Schlieren), for the cases with turbulent and laminar inflows, are shown in figure 11. A qualitative comparison reveals that the mixing layer with turbulent inflow has a smaller growth rate, consistent with observations reported in the literature.

Table 2. Comparison of experimental data from Slessor et al. ${ }^{1}$ and data from present simulations.

\begin{tabular}{ccclll} 
Run & $\delta_{T} / x$ & $\delta_{T} /(\mathrm{mm})$ & $\delta_{p} /(\mathrm{mm})$ & $\delta_{p} / \delta_{T}$ & $R e_{\delta} \times 10^{5}$ \\
\hline \hline Experiment, tripped & 0.140 & 51.1 & 15.0 & 0.293 & 1.8 \\
Present simulation & 0.144 & 56.8 & 17.0 & 0.299 & 2.0 \\
\hline
\end{tabular}

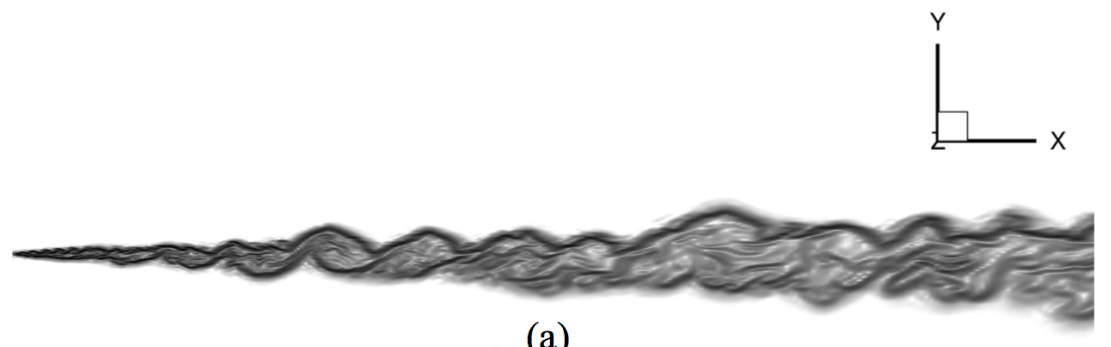

(a)

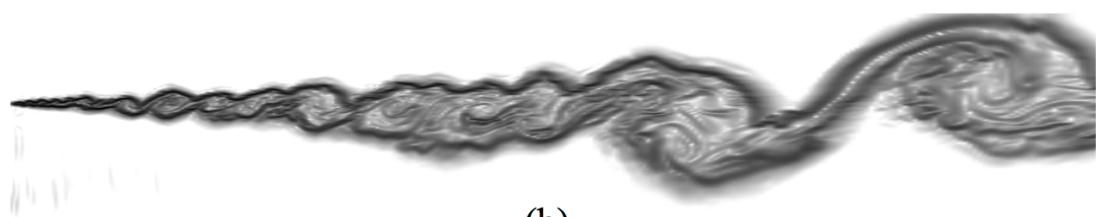

(b)

Figure 11. The comparison of the density gradient magnitude plot from the simulations with (a) turbulent inflow and (b) laminar inflow.

\section{Temperature profile}

For the high Damköhler number reactions, as in the case studied here, the temperature-rise is a direct indicator of molecular mixing. The temperature-rise normalized by the adiabatic flame temperature rise is plotted in figure 12(a). The temperature rise plotted is obtained by subtracting the free stream temperature from the temperature obtained in the chemically reacting flow simulation. The temperature-rise profile from the simulation is in good agreement with the experiment. The shape of the temperature profile and the spread is captured reasonably well, considering the coarse grids used. However, the location of the maximum temperature-rise is not accurately predicted. The comparison of temperature-rise profiles from Ferrero et al.(laminar inflow) is shown in figure 12. The comparison reveals that the error in the normalized maximum temperature-rise is about $10 \%$ which is significantly lower than the error in the prediction of temperature-rise $(27 \%)$ in the previous study. ${ }^{9}$ We believe numerical investigation of this flow field with different sub-grid scale models and a grid-refinement study will help us understand the error in the temperature-rise profiles this is currently underway. 


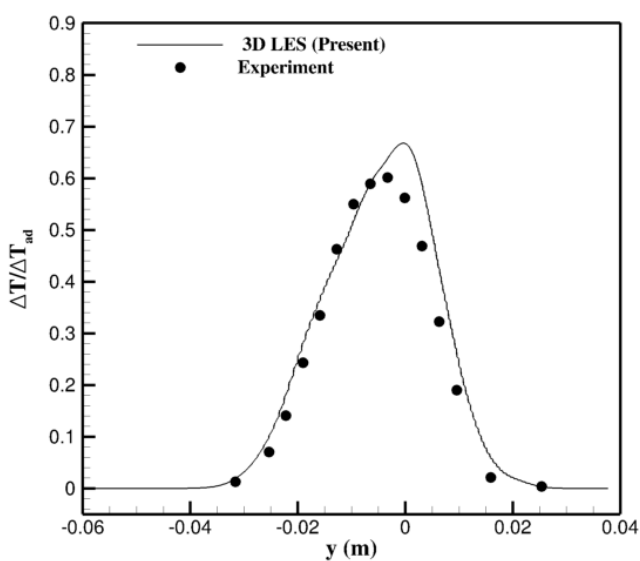

(a)

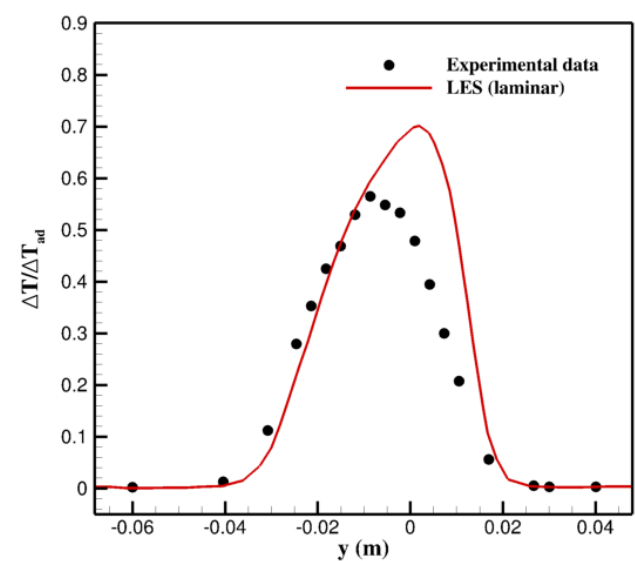

(b)

Figure 12. Temperature-rise profiles from (a) present simulation and (b) simulation in Ferrero et al. ${ }^{9}$ The data from the present simulation corresponds to a turbulent inflow condition and the data from Ferrero et al. corresponds to a laminar inflow condition.

\section{Mixture fraction}

"Numerical probes" were placed in the test section to collect statistics. We collect data from nine streamwise stations (evenly spaced between $x=0.10 \mathrm{~m}$ and $x=0.41 \mathrm{~m}$ ). At each of these stations, around 50 numerical probes were placed along the height of the test section and across the spanwise direction (20 probes in the span). The data collected was used to calculate the mixture fraction $\xi$, which is defined as the mole fraction of high-speed stream fluid. With this definition, $\xi=0$ corresponds to pure low-speed-stream fluid, $\xi=1$ represents pure high-speed-stream fluid.

In our preliminary analysis, we compare our data with results from the experiments of Pickett \& Ghandhi. ${ }^{6}$ As a caveat, we note that the Reynolds number in the experiment is a order of magnitude lower than the present work. Prior experimental work on passive scalar mixing in shear layers has revealed that the mean profiles (in the transverse direction) of $\xi$ vary smoothly, without inflection points, for mixing layer with turbulent inflow streams. We observe a similar trend in this work. This is shown in figure 13(a). Furthermore, our data and that of Pickett \& Ghandhi both produce asymmetric shapes for the $\xi_{r m s}$ profiles (figure 13(b)): the peak tilts towards the low-speed side of the mixing layer. The experimental data shows that the probability density function (PDF) of the mixture fraction changes its behavior from a 'marching' (most probable fluid composition varies linearly across the mixing layer thickness) to a 'distributed' shape (with no preference for any specific composition) at locations corresponding to peak $\xi_{\text {rms }}$. In figure 13(c), we observe that the nature of the PDF in the faster stream and in the lower stream is different, hinting at a hybrid-style PDF: further analysis is needed to confirm this.

\section{Conclusion}

In this work a chemically reacting, spatially evolving, high-Re mixing layer is studied using large-eddy simulations. The issue of spurious reactions and associated erroneous temperature rise resulting from excursions of species concentration is addressed by modifying the convective fluxes. Using these modified fluxes, simulations were performed which showed excellent agreement with the experimental data for velocity profiles at the measuring location. Also, the growth rate and the product thickness closely match the results obtained in the experiments. Mitigating the temperature excursions, with minimum numerical dissipation, remains a part of our ongoing effort to accurately simulate high-speed reacting flows via LES. The future work will focus on understanding the trends in the mixture fraction behavior in a chemically reacting, spatially evolving mixing layer. Effects of grid-refinement and SGS models will also be studied. 


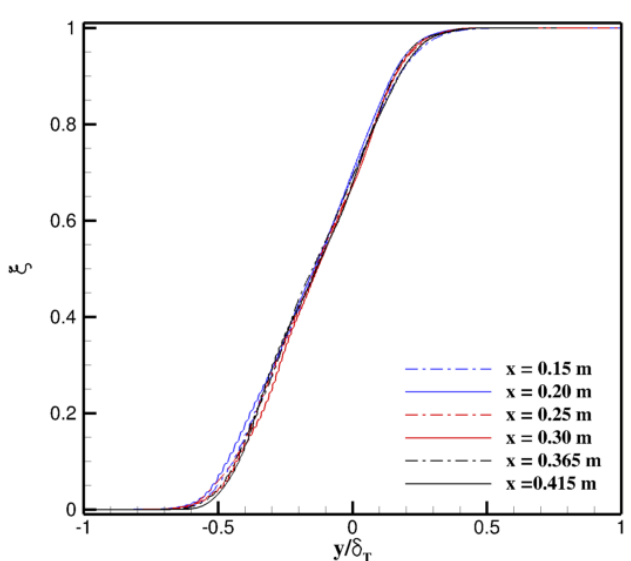

(a)

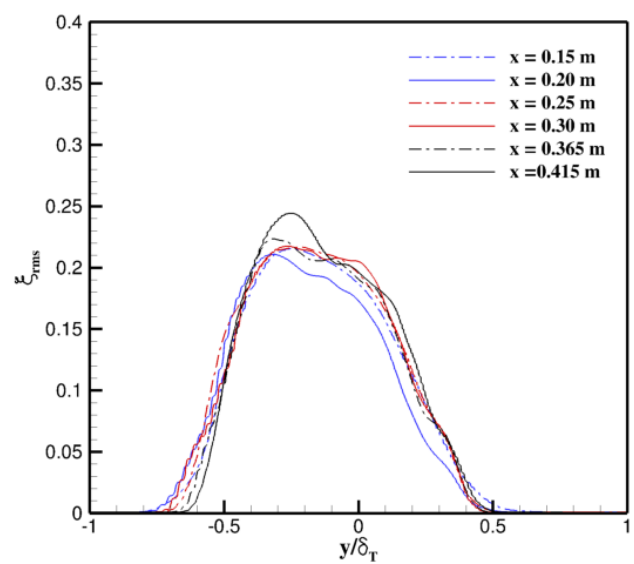

(b)

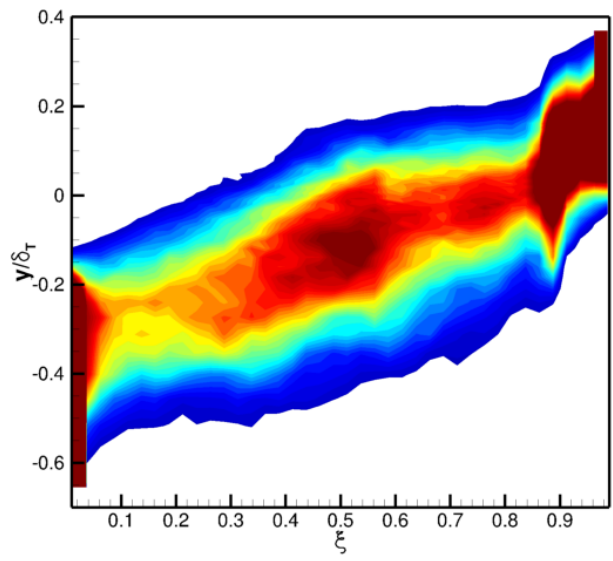

(c)

Figure 13. Plot of mixture fraction (a) mean, (b) variance at different streamwise locations and (c) probability density function of the mixture fraction.

\section{Acknowledgements}

This work was sponsored by the Air Force Office of Scientific Research under grants FA9550-12-1-0064 and FA9550-12-1-0461 and the Department of Energy. The views and conclusions contained herein are those of the author and should not be interpreted as necessarily representing the official policies or endorsements, either expressed or implied, of the AFOSR or the U.S. Government. This work was carried out in part using computing resources at the University of Minnesota Supercomputing Institute. We would also like to thank Sidharth G.S., Joesph Brock, Ross Choudary and Derek Dinzl for constructive discussions during the work.

\section{References}

${ }^{1}$ Slessor, M., Bond, C., and Dimotakis, P., "Turbulent shear-layer mixing at high Reynolds numbers: effects of inflow conditions," Journal of Fluid Mechanics, Vol. 376, 1998, pp. 115-138.

${ }^{2}$ Klein, M., Sadiki, A., and Janicka, J., "A digital filter based generation of inflow data for spatially developing direct numerical or large eddy simulations," Journal of computational Physics, Vol. 186, No. 2, 2003, pp. 652-665. 
${ }^{3}$ Liepmann, H. W. and Laufer, J., "Investigations of free turbulent mixing," 1947.

${ }^{4}$ Wygnanski, I. and Fiedler, H., "The two-dimensional mixing region," Journal of Fluid Mechanics, Vol. 41, No. 02, 1970, pp. $327-361$.

${ }^{5}$ Karasso, P. and Mungal, M., "Scalar mixing and reaction in plane liquid shear layers," Journal of Fluid Mechanics, Vol. 323, 1996, pp. 23-63.

${ }^{6}$ Pickett, L. M. and Ghandhi, J. B., "Passive scalar mixing in a planar shear layer with laminar and turbulent inlet conditions," Physics of Fluids, Vol. 14, No. 3, 2002, pp. 985-998.

${ }^{7}$ Matheou, G. and Dimotakis, P. E., "Scalar excursions in large-eddy simulations," AIAA Paper-3209, 2014.

${ }^{8}$ Subbareddy, P. K., Candler, G. V., and Ferrero, P., "Scalar conservation in Large Eddy Simulations of reacting flows," AIA A Paper-3203, 2014.

${ }^{9}$ Ferrero, P., Kartha, A., Subbareddy, P. K., Candler, G. V., and Dimotakis, P. E., "LES of a high-Reynolds number, chemically reacting mixing layer," AIAA Conference, 2013.

${ }^{10}$ Bradshaw, P., "The effect of initial conditions on the development of a free shear layer," Journal of Fluid Mechanics, Vol. 26, No. 02, 1966, pp. 225-236.

${ }^{11}$ BATT, R. G., "Layer Some Measurements on the Effect of Tripping the Two-Dimensional Shear," AIAA Journal, Vol. 13 , No. 2, 1975, pp. 245-247.

${ }^{12}$ Bell, J. H. and Mehta, R. D., "Development of a two-stream mixing layer from tripped and untripped boundary layers," AIA A journal, Vol. 28, No. 12, 1990, pp. 2034-2042.

${ }^{13}$ Oster, D., Wygnanski, I., and Fiedler, H., "Some preliminary observations on the effect of initial conditions on the structure of the two-dimensional turbulent mixing layer," Turbulence in internal flows.(ed. Murthy, SNB), 1977, pp. 67-87.

${ }^{14}$ Browand, F. and Latigo, B., "Growth of the two-dimensional mixing layer from a turbulent and nonturbulent boundary layer," Physics of Fluids (1958-1988), Vol. 22, No. 6, 1979, pp. 1011-1019.

${ }^{15}$ Mungal, M., Dimotakis, P., and Hermanson, J., "Reynolds number effects on mixing and combustion in a reacting shearlayer," AIAA journal, Vol. 23, No. 9, 1985, pp. 1418-1423.

${ }^{16}$ Rogers, M. M. and Moser, R. D., "Direct simulation of a self-similar turbulent mixing layer," Physics of Fluids, Vol. 6, No. 2, 1994, pp. 903-923.

${ }^{17}$ Ansari, A., "Self-similarity and mixing characteristics of turbulent mixing layers starting from laminar initial conditions," Physics of Fluids, Vol. 9, No. 6, 1997, pp. 1714-1728.

${ }^{18}$ Subbareddy, P., Peterson, D., Candler, G. V., and Marusic, I., "A synthetic inflow generation method using the attached eddy hypothesis," AIAA paper, Vol. 3672, 2006, pp. 2006.

${ }^{19} \mathrm{Li}, \mathrm{N}$., Balaras, E., and Piomelli, U., "Inflow conditions for large-eddy simulations of mixing layers," Physics of Fluids (1994-present), Vol. 12, No. 4, 2000, pp. 935-938.

${ }^{20}$ McMullan, W. and Garrett, S., "Streamwise Vortices in Plane Mixing Layers Originating from Laminar or Turbulent Initial Conditions," AIAA paper-1969, 2015.

${ }^{21}$ Nompelis, I., Drayna, T. W., and Candler, G. V., "A parallel unstructured implicit solver for hypersonic reacting flow simulation," AIAA paper, Vol. 4867, 2005.

${ }^{22}$ Subbareddy, P. K. and Candler, G. V., "A fully discrete, kinetic energy consistent finite-volume scheme for compressible flows," Journal of Computational Physics, Vol. 228, No. 5, 2009, pp. 1347-1364.

${ }^{23}$ Bartkowicz, M. D., Subbareddy, P. K., and Candler, G. V., "Numerical simulations of roughness induced instability in the Purdue Mach 6 wind tunnel," AIAA paper, Vol. 4723, 2010.

${ }^{24}$ Ducros, F., Ferrand, V., Nicoud, F., Weber, C., Darracq, D., Gacherieu, C., and Poinsot, T., "Large-eddy simulation of the shock/turbulence interaction," Journal of Computational Physics, Vol. 152, No. 2, 1999, pp. 517-549.

${ }^{25}$ Mavriplis, D. J., "Revisiting the least-squares procedure for gradient reconstruction on unstructured meshes," AIAA paper, Vol. 3986, 2003, pp. 2003.

${ }^{26} \mathrm{Shu}$, C.-W. and Osher, S., "Efficient implementation of essentially non-oscillatory shock-capturing schemes," Journal of Computational Physics, Vol. 77, No. 2, 1988, pp. 439-471.

${ }^{27}$ Zhang, X. and Shu, C.-W., "On maximum-principle-satisfying high order schemes for scalar conservation laws," Journal of Computational Physics, Vol. 229, No. 9, 2010, pp. 3091-3120.

${ }^{28}$ Poinsot, T. J. and Lele, S., "Boundary conditions for direct simulations of compressible viscous flows," Journal of computational physics, Vol. 101, No. 1, 1992, pp. 104-129.

${ }^{29}$ Xie, Z.-T. and Castro, I. P., "Efficient generation of inflow conditions for large eddy simulation of street-scale flows," Flow, turbulence and combustion, Vol. 81, No. 3, 2008, pp. 449-470.

${ }^{30}$ Touber, E. and Sandham, N. D., "Large-eddy simulation of low-frequency unsteadiness in a turbulent shock-induced separation bubble," Theoretical and Computational Fluid Dynamics, Vol. 23, No. 2, 2009, pp. 79-107.

${ }^{31}$ Yoo, C. S. and Im, H. G., "Characteristic boundary conditions for simulations of compressible reacting flows with multidimensional, viscous and reaction effects," Combustion Theory and Modelling, Vol. 11, No. 2, 2007, pp. 259-286.

${ }^{32}$ De Graaff, D. B. and Eaton, J. K., "Reynolds-number scaling of the flat-plate turbulent boundary layer," Journal of Fluid Mechanics, Vol. 422, 2000, pp. 319-346.

${ }^{33}$ Yee, H. C., Vinokur, M., and Djomehri, M., "Entropy splitting and numerical dissipation," Journal of Computational Physics, Vol. 162, No. 1, 2000, pp. 33-81. 